\title{
Convergence of a Class of Schrödinger Equations
}

\author{
Dan Li and Haixia Yu*
}

\begin{abstract}
In this paper, we set up the selection conditions for time series $\left\{t_{k}\right\}_{k=1}^{\infty}$ which converge to 0 as $k \rightarrow \infty$ such that the solutions of a class of generalized Schrödinger equations almost everywhere pointwise converge to their initial data in $H^{s}\left(\mathbb{R}^{n}\right)$ for $s>0$. As it is known that the pointwise convergence can not be true for Schrödinger equation when $s<\frac{n}{2(n+1)}$ as $t \rightarrow 0$.
\end{abstract}

\section{Introduction}

We first consider the a-th order Schrödinger equation defined on $\mathbb{R}^{n+1}$ such that

$$
\left\{\begin{aligned}
i u_{t}+(-\Delta)^{\frac{a}{2}} u & =0, & & t>0, \\
u(x, 0) & =f(x), & & t=0,
\end{aligned}\right.
$$

where $a \in(0, \infty)$ and $f \in H^{s}\left(\mathbb{R}^{n}\right)$. The Sobolev spaces $H^{s}\left(\mathbb{R}^{n}\right)$ is defined as

$$
H^{s}\left(\mathbb{R}^{n}\right):=\left\{f \in \mathcal{S}^{\prime}:\|f\|_{H^{s}\left(\mathbb{R}^{n}\right)}<\infty\right\}, \quad s \in \mathbb{R}
$$

with

$$
\|f\|_{H^{s}\left(\mathbb{R}^{n}\right)}:=\left(\int_{\mathbb{R}^{n}}\left(1+|\xi|^{2}\right)^{s}|\widehat{f}(\xi)|^{2} \mathrm{~d} \xi\right)^{\frac{1}{2}} .
$$

The solution of (1.1) can formally be written as

$$
u(x, t)=(2 \pi)^{-n} S_{t, a} f(x) .
$$

Here

$$
S_{t, a} f(x):=\int_{\mathbb{R}^{n}} e^{i x \cdot \xi} e^{i t|\xi|^{a}} \widehat{f}(\xi) \mathrm{d} \xi, \quad x \in \mathbb{R}^{n}, \quad t \geq 0,
$$

and

$$
\widehat{f}(\xi):=\int_{\mathbb{R}^{n}} e^{-i \xi \cdot x} f(x) \mathrm{d} x, \quad \xi \in \mathbb{R}^{n} .
$$

Carleson [4] proposed a problem that determine the optimal $s_{c}$ such that

$$
\lim _{t \rightarrow 0}(2 \pi)^{-n} S_{t, 2} f(x)=f(x) \quad \text { a.e. } x \in \mathbb{R}^{n}
$$

2010 Mathematics Subject Classification. Primary 35Q41.

Key words and phrases. Schrödinger equation, convergence, Sobolev spaces.

${ }^{*}$ Corresponding author. 
for all $f \in H^{s}\left(\mathbb{R}^{n}\right)$ with $s \geq s_{c}$. In the last four decades, Carleson Problem (1.3) has attracted numerous attentions (see [1, 2, 3, 10, 11, 13, 16, 18, 20, 22] and the referees therein). And now it is known that $s_{c}=\frac{n}{2(n+1)}$ for $n \geq 1$ is the critical index. For $n=1$, Carleson [4] set up (1.3) for $s \geq \frac{1}{4}$ and Dahlberg and Kenig [7] showed that (1.3) does not hold for $s<\frac{1}{4}$. For $n \geq 2$, Bourgain [3] formulated the counterexamples for all the case $s<\frac{n}{2(n+1)}$. Very recently, Du, Guth and Li [9] set up (1.3) for $s>\frac{1}{3}$ and $n=2$. Du and Zhang [12] then proved (1.3) for $s>\frac{n}{2(n+1)}$ and $n \geq 2$.

There are some results for the $a$-th order Schrödinger equation. If we replace $S_{t, 2}$ in (1.3) by $S_{t, a}$, we know that (1.3) holds for $f \in H^{\frac{1}{2}}\left(\mathbb{R}^{2}\right)$ in the case that $n=2$ and $a>1$. When $n \geq 3$ and $a>1$, (1.3) has been proved for $f \in H^{s}\left(\mathbb{R}^{n}\right)$ with $s>\frac{1}{2}$; see, for example, [18, 21]. Miao, Yang and Zheng [15] showed that (1.3) holds for $s>\frac{3}{8}$ when $n=2$ and $a>1$. Recently, Cho and Ko [5] improved this result to $s>\frac{n}{2(n+1)}$ when $n \geq 1$ and $a>1$.

On the other hand, it is easy to see that $\lim _{t \rightarrow 0}\left\|(2 \pi)^{-n} S_{t, a} f(x)-f(x)\right\|_{L^{2}\left(\mathbb{R}^{n}\right)}=0$. From Riesz theorem there exists $\left\{t_{k}\right\}_{k=1}^{\infty} \rightarrow 0$ as $k \rightarrow \infty$ such that

$$
\lim _{k \rightarrow \infty}(2 \pi)^{-n} S_{t_{k}, a} f(x)=f(x), \quad \text { a.e. } x \in \mathbb{R}^{n}
$$

for $s \geq 0$. In [19] Sjölin showed that (1.4] holds whenever $\sum_{k=1}^{\infty} t_{k} \frac{2 s}{a}<\infty$. In this paper we try to consider this kind of pointwise convergence for some generalized Schödinger equations. We first want to extend Sjölin's results to $0<a \leq 1$.

Theorem 1.1. Let $n \geq 1,0<a<1, s \geq a$. Assume that $\sum_{k=1}^{\infty} t_{k}^{2}<\infty, f \in H^{s}\left(\mathbb{R}^{n}\right)$. Then

$$
\lim _{k \rightarrow \infty}(2 \pi)^{-n} S_{t_{k}, a} f(x)=f(x)
$$

holds for almost everywhere $x \in \mathbb{R}^{n}$.

Theorem 1.2. Let $n \geq 1,0<s \leq a \leq 1$. Assume that $\sum_{k=1}^{\infty} t_{k}^{\frac{2 s}{a}}<\infty, f \in H^{s}\left(\mathbb{R}^{n}\right)$. Then

$$
\lim _{k \rightarrow \infty}(2 \pi)^{-n} S_{t_{k}, a} f(x)=f(x)
$$

holds for almost everywhere $x \in \mathbb{R}^{n}$.

A natural generalization of the pointwise convergence problem is to ask almost everywhere convergence along a wider approach region instead of vertical lines. One of such problems may be non-tangential convergence to the initial data for a.e. $x \in \mathbb{R}^{n}$. That is, for $b>0$ and $f \in H^{s}\left(\mathbb{R}^{n}\right)$, for which $s$ such that

$$
\lim _{(y, t) \in \Gamma_{b}(x),(y, t) \rightarrow(x, 0)}(2 \pi)^{-n} S_{t, 2} f(y)=f(x) \quad \text { a.e. } x \in \mathbb{R}^{n},
$$

where $\Gamma_{b}(x)=\left\{(y, t) \in \mathbb{R}_{+}^{n+1}:|y-x|<b t\right\}$. Sjögren and Sjölin [17] proved that (1.5]) fails for $s \leq \frac{n}{2}$. In fact, Sjögren and Sjölin [17] proved that there exists an $f \in H^{\frac{n}{2}}\left(\mathbb{R}^{n}\right)$ and a strictly increasing function $\Gamma$ with $\Gamma(0)=0$ such that for all $x \in \mathbb{R}^{n}$,

$$
\lim _{|x-y|<\Gamma(t), t>0,(y, t) \rightarrow(x, 0)}(2 \pi)^{-n}\left|S_{t, 2} f(y)\right|=\infty .
$$


Another problem is to consider the relation between the degree of the tangency and regularity when $(x, t)$ approaches to $(x, 0)$ tangentially. One of the model problems raised by Cho, Lee and Vargas [6] is

$$
\lim _{t \rightarrow 0} S_{t, 2} f(\Gamma(x, t))=f(x), \quad \text { a.e. } x \in \mathbb{R} .
$$

When $n=1$, here the curve $\Gamma(x, t)$ approaches $(x, 0)$ tangentially to the hyperplane $\{(x, t): t=0\}$. Cho, Lee and Vargas [6] set up (1.6) for $s>\max \left\{\frac{1}{2}-\alpha, \frac{1}{4}\right\}$, here $\Gamma(x, t)$ satisfies Hölder condition of order $\alpha$ in $t$ and Bilipschitz condition in $x$ with $0<\alpha \leq 1 . \alpha$ is essentially the degree of tangential convergence. Ding and Niu [8] improved the result of Cho, Lee and Vargas [6] to $s \geq \frac{1}{4}$ when $n=1$ and $\frac{1}{2} \leq \alpha \leq 1$, but the problem is still open for $n \geq 2$. Recently, Li and Wang [14] proved convergence for $s>\frac{3}{8}$ when $n=2$ and $\Gamma(x, t):=x-\sqrt{t} \mu$, where $\mu$ is a unit vector in $\mathbb{R}^{2}$. Next we consider the general case and define

$$
S_{t, a} f\left(x+t^{\beta} \mu\right):=\int_{\mathbb{R}^{n}} e^{i\left(x \cdot \xi+t^{\beta} \mu \cdot \xi\right)} e^{i t|\xi|^{a}} \widehat{f}(\xi) \mathrm{d} \xi, \quad t \geq 0, \quad a>0, \quad \beta \in \mathbb{R},
$$

where $\mu$ is a unit vector in $\mathbb{R}^{n}$. For $S_{t, a} f\left(x+t^{\beta} \mu\right)$, we have that

Theorem 1.3. Let $n \geq 1,0<a<1,0<s \leq 1, s>1-a$ when $\beta>1$ and $s>1-a \beta$ when $\beta \leq 1$. Assume that $\sum_{k=1}^{\infty} t_{k}{ }^{2\left(1+\frac{s-1}{a}\right)}<\infty$ when $\beta>1, \sum_{k=1}^{\infty} t_{k}{ }^{2\left(\beta+\frac{s-1}{a}\right)}<\infty$ when $\beta \leq 1, f \in H^{s}\left(\mathbb{R}^{n}\right)$. Then

$$
\lim _{k \rightarrow \infty}(2 \pi)^{-n} S_{t_{k}, a} f\left(x+t^{\beta} \mu\right)=f(x)
$$

holds for almost everywhere $x \in \mathbb{R}^{n}$.

Theorem 1.4. Let $n \geq 1, a \geq 1,0<s \leq a$, $s>a(1-\beta)$ when $\beta \leq 1$. Assume that $\sum_{k=1}^{\infty} t_{k} \frac{2 s}{a}<\infty$ when $\beta>1, \sum_{k=1}^{\infty} t_{k}^{2\left(\beta-1+\frac{s}{a}\right)}<\infty$ when $\beta \leq 1, f \in H^{s}\left(\mathbb{R}^{n}\right)$. Then

$$
\lim _{k \rightarrow \infty}(2 \pi)^{-n} S_{t_{k}, a} f\left(x+t^{\beta} \mu\right)=f(x)
$$

holds for almost everywhere $x \in \mathbb{R}^{n}$.

The last part of this paper is to discuss the convergence of a class of dispersive equations

$$
\left\{\begin{aligned}
i u_{t}+\gamma(\sqrt{-\Delta}) u & =0, & & t>0, \\
u(x, 0) & =f(x), & & t=0,
\end{aligned}\right.
$$

where $\gamma: \mathbb{R}^{+} \rightarrow \mathbb{R}^{+}$is smooth, $f \in H^{s}\left(\mathbb{R}^{n}\right)$ with $n \geq 1$. The solution of (1.8) can formally be written as

$$
S_{t, \gamma} f(x):=\int_{\mathbb{R}^{n}} e^{i x \cdot \xi} e^{i t \gamma(\xi \xi)} \widehat{f}(\xi) \mathrm{d} \xi, \quad t \geq 0 .
$$

Next we consider the following pointwise convergence

$$
\lim _{k \rightarrow \infty}(2 \pi)^{-n} S_{t_{k}, \gamma} f(x)=f(x) \quad \text { a.e. } x \in \mathbb{R}^{n} .
$$

Very recently, under some assumptions on $\gamma(|\xi|)$ Cho and Ko [5] set up (1.10) whenever $s>\frac{n}{2(n+1)}$. For example, $\gamma(|\xi|)=|\xi|^{a}$ with $a>1$. These assumptions also appeared in [2] and [13]. For $S_{t, \gamma}$, we have that 
Theorem 1.5. Let $n \geq 1,0<s \leq 1, \gamma(t) \geq 0$ and $\frac{\gamma(t)}{t}$ be smooth and increasing on $(0,+\infty)$. Assume that $\sum_{k=1}^{\infty} \frac{1}{\left[\gamma^{-1}\left(\frac{\gamma(1)}{t_{k}}\right)\right]^{2 s}}<\infty, f \in H^{s}\left(\mathbb{R}^{n}\right)$. Then

$$
\lim _{k \rightarrow \infty}(2 \pi)^{-n} S_{t_{k}, \gamma} f(x)=f(x)
$$

holds for almost everywhere $x \in \mathbb{R}^{n}$, where $\gamma^{-1}$ is the inverse function of $\gamma$.

Remark 1.6. If we take $\gamma(|\xi|):=|\xi|^{a}$ with $a>1$, we come back to Theorem 1 in Sjölin [19]. Further more, if we take $\gamma(|\xi|):=|\xi|$, Theorem 1.5 does also hold. If we take $\gamma(|\xi|):=|\xi| \sqrt{1+|\xi|^{2}}$, we have the Boussinesq equation which is defined on $\mathbb{R}^{n+1}$ such that

$$
\left\{\begin{aligned}
i u_{t}+\sqrt{-\Delta} \sqrt{1-\Delta} u & =0, & & t>0, \\
u(x, 0) & =f(x), & & t=0,
\end{aligned}\right.
$$

for all $f \in H^{s}\left(\mathbb{R}^{n}\right)$. Its solution can formally be written as

$$
S_{t, \sqrt{-\Delta} \sqrt{1-\Delta}} f(x):=\int_{\mathbb{R}^{n}} e^{i x \cdot \xi} e^{i t|\xi|} \sqrt{1+|\xi|^{2}} \widehat{f}(\xi) \mathrm{d} \xi, \quad t \geq 0 .
$$

From Theorem 1.5, we have the following conclusion. Let $n \geq 1,0<s \leq 1$. Assume that $\sum_{k=1}^{\infty} t_{k}^{s}<\infty, f \in H^{s}\left(\mathbb{R}^{n}\right)$. Then

$$
\lim _{k \rightarrow \infty}(2 \pi)^{-n} S_{t_{k}, \sqrt{-\Delta} \sqrt{1-\Delta}} f(x)=f(x)
$$

holds for almost everywhere $x \in \mathbb{R}^{n}$. If we take $\gamma(|\xi|):=|\xi|^{2}+|\xi|^{4}$, we have the 4-order Schrödinger equation which is defined on $\mathbb{R}^{n+1}$ such that

$$
\left\{\begin{aligned}
i u_{t}+\left[(-\Delta)+(-\Delta)^{2}\right] u & =0, & & t>0, \\
u(x, 0) & =f(x), & t & =0,
\end{aligned}\right.
$$

for all $f \in H^{s}\left(\mathbb{R}^{n}\right)$. Its solution can formally be written as

$$
S_{t,(-\Delta)+(-\Delta)^{2}} f(x):=\int_{\mathbb{R}^{n}} e^{i x \cdot \xi} e^{i t\left(|\xi|^{2}+|\xi|^{4}\right)} \widehat{f}(\xi) \mathrm{d} \xi, \quad t \geq 0 .
$$

From Theorem 1.5, we have the following result. Let $n \geq 1,0<s \leq 1$. Assume that $\sum_{k=1}^{\infty} t_{k}^{\frac{s}{2}}<\infty$, $f \in H^{s}\left(\mathbb{R}^{n}\right)$. Then

$$
\lim _{k \rightarrow \infty}(2 \pi)^{-n} S_{t,(-\Delta)+(-\Delta)^{2}} f(x)=f(x)
$$

holds for almost everywhere $x \in \mathbb{R}^{n}$.

Finally, we want to extend the Theorem 1.3 and Theorem 1.4 to general case, so we define

$$
S_{t, \gamma} f\left(x+t^{\beta} \mu\right):=\int_{\mathbb{R}^{n}} e^{i\left(x \cdot \xi+t^{\beta} \mu \cdot \xi\right)} e^{i t \gamma(|\xi|)} \widehat{f}(\xi) \mathrm{d} \xi, \quad t \geq 0, \quad \beta \in \mathbb{R},
$$

where $\mu$ is a unit vector in $\mathbb{R}^{n}$. Next we consider the following pointwise convergence

$$
\lim _{k \rightarrow \infty}(2 \pi)^{-n} S_{t, \gamma} f\left(x+t^{\beta} \mu\right)=f(x) \quad \text { a.e. } x \in \mathbb{R}^{n} .
$$

For $S_{t, \gamma} f\left(x+t^{\beta} \mu\right)$, we have that 
Theorem 1.7. Let $n \geq 1,0<s \leq 1, \gamma(t)$ be the same as in Theorem 1.5 Assume that $\sum_{k=1}^{\infty} \frac{1}{\left[\gamma^{-1}\left(\frac{\gamma(1)}{t_{k}}\right)\right]^{2 s}}<\infty$ when $\beta>1$ and $\sum_{k=1}^{\infty} \frac{t_{k}^{\beta-1}}{\left[\gamma^{-1}\left(\frac{\gamma(1)}{t_{k}}\right)\right]^{2 s}}<\infty$ when $\beta \leq 1, f \in H^{s}\left(\mathbb{R}^{n}\right)$. Then

$$
\lim _{k \rightarrow \infty}(2 \pi)^{-n} S_{t_{k}, \gamma} f\left(x+t^{\beta} \mu\right)=f(x)
$$

holds for almost everywhere $x \in \mathbb{R}^{n}$, where $\gamma^{-1}$ is the inverse function of $\gamma$.

Throughout this paper, we use $C$ to denote a positive constant which is independent of the essential variables but its value may be different from line to line. $f \lesssim g$ means $f \leq C g$ and $f \approx g$ means $f \lesssim g \lesssim f$.

\section{Proof of the main results}

Proof of Theorem 1.1 Without loss of generality we can assume $0 \leq t_{k}<1$. Let $g \in \mathcal{S}\left(\mathbb{R}^{n}\right)$, so

$$
(2 \pi)^{-n} S_{t_{k}, a} g(x)=(2 \pi)^{-n} \int_{\mathbb{R}^{n}} e^{i x \cdot \xi} e^{i t_{k}|\xi|^{a}} \widehat{g}(\xi) \mathrm{d} \xi
$$

Since

$$
g(x)=(2 \pi)^{-n} \int_{\mathbb{R}^{n}} e^{i x \cdot \xi} \widehat{g}(\xi) \mathrm{d} \xi
$$

Then

$$
(2 \pi)^{-n} S_{t_{k}, a} g(x)-g(x)=(2 \pi)^{-n} \int_{\mathbb{R}^{n}} e^{i x \cdot \xi}\left(e^{i t_{k}|\xi|^{a}}-1\right) \widehat{g}(\xi) \mathrm{d} \xi .
$$

We define $\widehat{\varphi}(\xi):=\left(1+|\xi|^{2}\right)^{\frac{s}{2}} \widehat{g}(\xi)$. Then

$$
\begin{aligned}
(2 \pi)^{-n} S_{t_{k}, a} g(x)-g(x) & =(2 \pi)^{-n} \int_{\mathbb{R}^{n}} e^{i x \cdot \xi}\left(e^{i t_{k}|\xi|^{a}}-1\right)\left(1+|\xi|^{2}\right)^{-\frac{s}{2}} \widehat{\varphi}(\xi) \mathrm{d} \xi \\
& =(2 \pi)^{-n} \int_{\mathbb{R}^{n}} e^{i x \cdot \xi} m_{a}(\xi) \widehat{\varphi}(\xi) \mathrm{d} \xi
\end{aligned}
$$

where $m_{a}(\xi)=\frac{e^{i t_{k}|\xi|^{a}}-1}{\left(1+|\xi|^{2}\right)^{\frac{s}{2}}}$. Then

$$
\left|m_{a}(\xi)\right|=\left|\frac{e^{i t_{k}|\xi|^{a}}-1}{\left(1+|\xi|^{2}\right)^{\frac{s}{2}}}\right| \lesssim \frac{t_{k}|\xi|^{a}}{\left(1+|\xi|^{2}\right)^{\frac{s}{2}}} \leq t_{k}
$$

for $a \leq s$. We obtain $\left\|m_{a}\right\|_{L^{\infty}\left(\mathbb{R}^{n}\right)} \lesssim t_{k}$. According to Plancherel's theorem we can get

$$
\left\|(2 \pi)^{-n} S_{t_{k}, a} g(x)-g(x)\right\|_{L^{2}\left(\mathbb{R}^{n}\right)}^{2}=\left\|m_{a} \widehat{\varphi}\right\|_{L^{2}\left(\mathbb{R}^{n}\right)}^{2} \leq\left\|m_{a}\right\|_{L^{\infty}\left(\mathbb{R}^{n}\right)}^{2}\|\widehat{\varphi}\|_{L^{2}\left(\mathbb{R}^{n}\right)}^{2} \lesssim t_{k}^{2}\|g\|_{H^{s}\left(\mathbb{R}^{n}\right)}^{2} .
$$

So far we have proved that

$$
\left\|(2 \pi)^{-n} S_{t_{k}, a} g(x)-g(x)\right\|_{L^{2}\left(\mathbb{R}^{n}\right)} \lesssim t_{k}\|g\|_{H^{s}\left(\mathbb{R}^{n}\right)}, \quad g \in \mathcal{S}\left(\mathbb{R}^{n}\right) .
$$


Next we want to prove

$$
\left\|h_{k, a}\right\|_{L^{2}\left(\mathbb{R}^{n}\right)}=\left\|(2 \pi)^{-n} S_{t_{k}, a} f(x)-f(x)\right\|_{L^{2}\left(\mathbb{R}^{n}\right)} \lesssim t_{k}\|f\|_{H^{s}\left(\mathbb{R}^{n}\right)}, \quad f \in H^{s}\left(\mathbb{R}^{n}\right),
$$

where $h_{k, a}(x)=(2 \pi)^{-n} S_{t_{k}, a} f(x)-f(x)$. Since the Schwartz space $\mathcal{S}\left(\mathbb{R}^{n}\right)$ is dense in $H^{s}\left(\mathbb{R}^{n}\right)$, so for any function $f \in H^{s}\left(\mathbb{R}^{n}\right)$, we can find $g_{k} \in \mathcal{S}\left(\mathbb{R}^{n}\right)$ satisfying that

$$
\left\|g_{k}-f\right\|_{H^{s}\left(\mathbb{R}^{n}\right)} \leq \frac{1}{100} t_{k}\|f\|_{H^{s}\left(\mathbb{R}^{n}\right)}
$$

This implies that

$$
\begin{aligned}
\left\|h_{k, a}\right\|_{L^{2}\left(\mathbb{R}^{n}\right)} & =\left\|(2 \pi)^{-n} S_{t_{k}, a} f(x)-f(x)\right\|_{L^{2}\left(\mathbb{R}^{n}\right)} \\
& \leq\left\|(2 \pi)^{-n} S_{t_{k}, a}\left(f-g_{k}\right)(x)\right\|_{L^{2}\left(\mathbb{R}^{n}\right)}+\left\|(2 \pi)^{-n} S_{t_{k}, a}\left(g_{k}\right)(x)-g_{k}(x)\right\|_{L^{2}\left(\mathbb{R}^{n}\right)}+\left\|g_{k}(x)-f(x)\right\|_{L^{2}\left(\mathbb{R}^{n}\right)} \\
& \lesssim\left\|f-g_{k}\right\|_{L^{2}\left(\mathbb{R}^{n}\right)}+t_{k}\left\|g_{k}\right\|_{H^{s}\left(\mathbb{R}^{n}\right)}+\left\|f-g_{k}\right\|_{L^{2}\left(\mathbb{R}^{n}\right)} \\
& \lesssim\left\|f-g_{k}\right\|_{H^{s}\left(\mathbb{R}^{n}\right)}+t_{k}\left(\left\|f-g_{k}\right\|_{H^{s}\left(\mathbb{R}^{n}\right)}+\|f\|_{H^{s}\left(\mathbb{R}^{n}\right)}\right) \\
& \leq \frac{1}{100} t_{k}\|f\|_{H^{s}\left(\mathbb{R}^{n}\right)}+\frac{1}{100} t_{k}^{2}\|f\|_{H^{s}\left(\mathbb{R}^{n}\right)}+t_{k}\|f\|_{H^{s}\left(\mathbb{R}^{n}\right)} \\
& \lesssim t_{k}\|f\|_{H^{s}\left(\mathbb{R}^{n}\right)} .
\end{aligned}
$$

It follows that

$$
\sum_{k=1}^{\infty} \int_{\mathbb{R}^{n}}\left|h_{k, a}\right|^{2} \mathrm{~d} x \lesssim \sum_{k=1}^{\infty} t_{k}^{2}\|f\|_{H^{s}\left(\mathbb{R}^{n}\right)}^{2}<\infty .
$$

By Fubini's theorem we have $\int_{\mathbb{R}^{n}} \sum_{k=1}^{\infty}\left|h_{k, a}\right|^{2} \mathrm{~d} x<\infty$, which further implies that $\sum_{k=1}^{\infty}\left|h_{k, a}\right|^{2}<\infty$, a.e. $x \in \mathbb{R}^{n}$. Hence $\lim _{k \rightarrow \infty} h_{k, a}(x)=0$, a.e. $x \in \mathbb{R}^{n}$. Therefore,

$$
\lim _{k \rightarrow \infty}(2 \pi)^{-n} S_{t_{k}, a} f(x)=f(x)
$$

for almost everywhere $x \in \mathbb{R}^{n}$. This completes the proof of Theorem 1.1

The proof of Theorem 1.2 relies on the following lemma and Sjölin [19] considered the case of $a>1$. Here we assume $0<s \leq a \leq 1$.

Lemma 2.1. Let $n \geq 1,0<s \leq a \leq 1,0<\delta<1$ and $m_{a}(\xi)=\frac{e^{i \delta|\xi| a^{a}}-1}{\left(1+|\xi|^{2}\right)^{\frac{s}{2}}}$. Then $\left\|m_{a}\right\|_{L^{\infty}\left(\mathbb{R}^{n}\right)} \leq C \delta^{\frac{s}{a}}$, where the constant $C$ does not depend on $\delta$.

Proof. The proof can be divided into three situations.

(i) if $0 \leq|\xi| \leq 1$, we have

$$
\left|m_{a}(\xi)\right|=\left|\frac{e^{i \delta|\xi|^{a}}-1}{\left(1+|\xi|^{2}\right)^{\frac{s}{2}}}\right| \leq \delta|\xi|^{a} \leq \delta \leq \delta^{\frac{s}{a}}
$$

(ii) if $1<|\xi|<\delta^{-\frac{1}{a}}$, we obtain

$$
\left|m_{a}(\xi)\right| \leq \frac{\delta|\xi|^{a}}{|\xi|^{s}}=\delta|\xi|^{a-s} \leq \delta \delta^{-\frac{a-s}{a}}=\delta^{\frac{s}{a}}
$$


(iii) if $|\xi| \geq \delta^{-\frac{1}{a}}$, we get

$$
\left|m_{a}(\xi)\right| \lesssim \frac{1}{|\xi|^{s}} \leq \frac{1}{\delta^{-\frac{s}{a}}}=\delta^{\frac{s}{a}}
$$

Altogether we have shown that $\left\|m_{a}\right\|_{L^{\infty}\left(\mathbb{R}^{n}\right)} \lesssim \delta^{\frac{s}{a}}$, which completes the proof of Lemma 2.1

Proof of Theorem 1.2 Similarly to the proof of Theorem 1.1, we have that

$$
h_{k, a}(x)=(2 \pi)^{-n} \int_{\mathbb{R}^{n}} e^{i x \cdot \xi} m_{a}(\xi) \widehat{\varphi_{1}}(\xi) \mathrm{d} \xi
$$

From Lemma 2.1 we obtain $\left\|m_{a}\right\|_{L^{\infty}\left(\mathbb{R}^{n}\right)} \lesssim t_{k} \frac{s}{a}$. We may conclude that

$$
\left\|h_{k, a}\right\|_{L^{2}\left(\mathbb{R}^{n}\right)}^{2} \lesssim t_{k}^{\frac{2 s}{a}}\|f\|_{H^{s}\left(\mathbb{R}^{n}\right)}^{2}
$$

This, combined with the fact that $\sum_{k=1}^{\infty} t_{k}^{\frac{2 s}{a}}<\infty$, we assert that $\sum_{k=1}^{\infty} \int_{\mathbb{R}^{n}}\left|h_{k, a}\right|^{2} \mathrm{~d} x<\infty$. Applying Fubini's theorem again, we have $\int_{\mathbb{R}^{n}} \sum_{k=1}^{\infty}\left|h_{k, a}\right|^{2} \mathrm{~d} x<\infty$, which further implies that $\lim _{k \rightarrow \infty} h_{k, a}(x)=$ 0 , a.e. $x \in \mathbb{R}^{n}$. Therefore,

$$
\lim _{k \rightarrow \infty}(2 \pi)^{-n} S_{t_{k}, a} f(x)=f(x)
$$

for almost everywhere $x \in \mathbb{R}^{n}$. This completes the proof of Theorem 1.2 ,

Proof of Theorem 1.3 Similarly to the proof of Theorem 1.1 and Theorem 1.2, it suffices to show that $\left\|m_{a, \mu}\right\|_{L^{\infty}\left(\mathbb{R}^{n}\right)} \lesssim \delta^{1+\frac{s-1}{a}}$ when $\beta>1$ and $\left\|m_{a, \mu}\right\|_{L^{\infty}\left(\mathbb{R}^{n}\right)} \lesssim \delta^{\beta+\frac{s-1}{a}}$ when $\beta \leq 1$ for $m_{a, \mu}(\xi):=$ $\frac{e^{i\left(\delta \delta^{\beta} \mu \cdot \xi+\delta|\xi|^{a}\right)}-1}{\left(1+|\xi|^{2}\right)^{\frac{s}{2}}}$.

In fact, when $\beta>1$,

(i) if $0 \leq|\xi| \leq 1$, we have

$$
\left|m_{a, \mu}(\xi)\right|=\left|\frac{e^{i\left(\delta^{\beta} \mu \cdot \xi+\delta|\xi|^{a}\right)}-1}{\left(1+|\xi|^{2}\right)^{\frac{s}{2}}}\right| \leq\left.\left.\left|\delta^{\beta} \mu \cdot \xi+\delta\right| \xi\right|^{a}\left|\leq \delta\left(|\xi|+|\xi|^{a}\right) \leq 2 \delta\right| \xi\right|^{a} \leq 2 \delta \leq 2 \delta^{1+\frac{s-1}{a}} .
$$

(ii) if $1<|\xi|<\delta^{-\frac{1}{a}}$, we obtain

$$
\left|m_{a, \mu}(\xi)\right| \leq \frac{\left.\left|\delta^{\beta} \mu \cdot \xi+\delta\right| \xi\right|^{a} \mid}{|\xi|^{s}} \leq \frac{\delta\left(|\xi|+|\xi|^{a}\right)}{|\xi|^{s}} \leq \frac{2 \delta|\xi|}{|\xi|^{s}}=2 \delta|\xi|^{1-s} \leq 2 \delta \delta^{-\frac{1-s}{a}}=2 \delta^{1+\frac{s-1}{a}}
$$

(iii) if $|\xi| \geq \delta^{-\frac{1}{a}}$, we get

$$
\left|m_{a, \mu}(\xi)\right| \lesssim \frac{1}{|\xi|^{s}} \leq \frac{1}{\delta^{-\frac{s}{a}}} \leq \delta^{1+\frac{s-1}{a}}
$$

All of these imply that $\left\|m_{a, \mu}\right\|_{L^{\infty}\left(\mathbb{R}^{n}\right)} \lesssim \delta^{1+\frac{s-1}{a}}$ when $\beta>1$.

Next we consider the case of $\beta \leq 1$,

(i) if $0 \leq|\xi| \leq 1$, we have

$$
\left|m_{a, \mu}(\xi)\right|=\left|\frac{e^{i\left(\delta^{\beta} \mu \cdot \xi+\delta|\xi|^{a}\right)}-1}{\left(1+|\xi|^{2}\right)^{\frac{s}{2}}}\right| \leq\left.\left.\left|\delta^{\beta} \mu \cdot \xi+\delta\right| \xi\right|^{a}\left|\leq \delta^{\beta}\left(|\xi|+|\xi|^{a}\right) \leq 2 \delta^{\beta}\right| \xi\right|^{a} \leq 2 \delta^{\beta} \leq 2 \delta^{\beta+\frac{s-1}{a}}
$$


(ii) if $1<|\xi|<\delta^{-\frac{1}{a}}$, we obtain

$$
\left|m_{a, \mu}(\xi)\right| \leq \frac{\left.\left|\delta^{\beta} \mu \cdot \xi+\delta\right| \xi\right|^{a} \mid}{|\xi|^{s}} \leq \frac{\delta^{\beta}\left(|\xi|+|\xi|^{a}\right)}{|\xi|^{s}} \leq \frac{2 \delta^{\beta}|\xi|}{|\xi|^{s}}=2 \delta^{\beta}|\xi|^{1-s} \leq 2 \delta^{\beta} \delta^{-\frac{1-s}{a}}=2 \delta^{\beta+\frac{s-1}{a}}
$$

(iii) if $|\xi| \geq \delta^{-\frac{1}{a}}$, we get

$$
\left|m_{a, \mu}(\xi)\right| \lesssim \frac{1}{|\xi|^{s}} \leq \frac{1}{\delta^{-\frac{s}{a}}} \leq \delta^{\beta+\frac{s-1}{a}}
$$

This completes the proof of Theorem 1.3 .

Proof of Theorem 1.4 Similarly to the proof of Theorem 1.3, it is enough to show that $\left\|m_{a, \mu}\right\|_{L^{\infty}\left(\mathbb{R}^{n}\right)} \lesssim$ $\delta^{\frac{s}{a}}$ when $\beta>1$ and $\left\|m_{a, \mu}\right\|_{L^{\infty}\left(\mathbb{R}^{n}\right)} \lesssim \delta^{\beta-1+\frac{s}{a}}$ when $\beta \leq 1$.

When $\beta>1$,

(i) if $0 \leq|\xi| \leq 1$, we have

$$
\left|m_{a, \mu}(\xi)\right|=\left|\frac{e^{i\left(\delta^{\beta} \mu \cdot \xi+\delta|\xi|^{a}\right)}-1}{\left(1+|\xi|^{2}\right)^{\frac{s}{2}}}\right| \leq\left.\left|\delta^{\beta} \mu \cdot \xi+\delta\right| \xi\right|^{a}\left|\leq \delta\left(|\xi|+|\xi|^{a}\right) \leq 2 \delta\right| \xi \mid \leq 2 \delta \leq 2 \delta^{\frac{s}{a}} .
$$

(ii) if $1<|\xi|<\delta^{-\frac{1}{a}}$, we obtain

$$
\left|m_{a, \mu}(\xi)\right| \leq \frac{\left.\left|\delta^{\beta} \mu \cdot \xi+\delta\right| \xi\right|^{a} \mid}{|\xi|^{s}} \leq \frac{\delta\left(|\xi|+|\xi|^{a}\right)}{|\xi|^{s}} \leq \frac{2 \delta|\xi|^{a}}{|\xi|^{s}}=2 \delta|\xi|^{a-s} \leq 2 \delta \delta^{-\frac{a-s}{a}}=2 \delta^{\frac{s}{a}}
$$

(iii) if $|\xi| \geq \delta^{-\frac{1}{a}}$, we get

$$
\left|m_{a, \mu}(\xi)\right| \lesssim \frac{1}{|\xi|^{s}} \leq \frac{1}{\delta^{-\frac{s}{a}}}=\delta^{\frac{s}{a}}
$$

When $\beta \leq 1$,

(i) if $0 \leq|\xi| \leq 1$, we have

$$
\left|m_{a, \mu}(\xi)\right|=\left|\frac{e^{i\left(\delta^{\beta} \mu \cdot \xi+\delta|\xi|^{a}\right)}-1}{\left(1+|\xi|^{2}\right)^{\frac{s}{2}}}\right| \leq\left.\left|\delta^{\beta} \mu \cdot \xi+\delta\right| \xi\right|^{a}\left|\leq \delta^{\beta}\left(|\xi|+|\xi|^{a}\right) \leq 2 \delta^{\beta}\right| \xi \mid \leq 2 \delta^{\beta} \leq 2 \delta^{\beta-1+\frac{s}{a}}
$$

(ii) if $1<|\xi|<\delta^{-\frac{1}{a}}$, we obtain

$$
\left|m_{a, \mu}(\xi)\right| \leq \frac{\left.\left|\delta^{\beta} \mu \cdot \xi+\delta\right| \xi\right|^{a} \mid}{|\xi|^{s}} \leq \frac{\delta^{\beta}\left(|\xi|+|\xi|^{a}\right)}{|\xi|^{s}} \leq \frac{2 \delta^{\beta}|\xi|^{a}}{|\xi|^{s}}=2 \delta^{\beta}|\xi|^{a-s} \leq 2 \delta^{\beta} \delta^{-\frac{a-s}{a}}=2 \delta^{\beta-1+\frac{s}{a}}
$$

(iii) if $|\xi| \geq \delta^{-\frac{1}{a}}$, we get

$$
\left|m_{a, \mu}(\xi)\right| \lesssim \frac{1}{|\xi|^{s}} \leq \frac{1}{\delta^{-\frac{s}{a}}}=\delta^{\frac{s}{a}} \leq \delta^{\beta-1+\frac{s}{a}} .
$$

This completes the proof of Theorem 1.4 
In the proof of Theorem 1.5 , it suffices to proof the Lemma 2.2

Lemma 2.2. Let $n \geq 1,0<s \leq 1,0<\delta<1, m_{\gamma}(\xi):=\frac{e^{i \delta \gamma(\xi \xi)}-1}{\left(1+|\xi|^{2}\right)^{\frac{s}{2}}}, \gamma(t)$ be the same as in Theorem 1.5 We have $\left\|m_{\gamma}\right\|_{L^{\infty}\left(\mathbb{R}^{n}\right)} \leq C \frac{1}{\left[\gamma^{-1}\left(\frac{\gamma(1)}{\delta}\right)\right]^{s}}$, where the constant $C$ does not depend on $\delta$.

Proof. The proof can also be divided into three situations.

(i) if $0 \leq|\xi| \leq 1$, from the fact that $0<\delta<1$ and $\frac{\gamma(t)}{t}: \mathbb{R}^{+} \rightarrow \mathbb{R}^{+}$is smooth and increasing on $(0, \infty)$, we have that $\frac{\gamma(1)}{\delta} \leq \gamma\left(\frac{1}{\delta}\right)$. Since $\gamma(t): \mathbb{R}^{+} \rightarrow \mathbb{R}^{+}$is smooth and increasing on $(0, \infty)$, we further obtain that $\gamma^{-1}\left(\frac{\gamma(1)}{\delta}\right) \leq \frac{1}{\delta}$. By $0<s \leq 1$ and $0<\delta<1$, we assert that $\left[\gamma^{-1}\left(\frac{\gamma(1)}{\delta}\right)\right]^{s} \leq\left[\frac{1}{\delta}\right]^{s} \leq \frac{1}{\delta}$. Consequently, we may conclude that $\delta \leq \frac{1}{\left[\gamma^{-1}\left(\frac{\gamma(1)}{\delta}\right)\right]^{s}}$. The above implies

$$
\left|m_{\gamma}(\xi)\right|=\left|\frac{e^{i \delta \gamma(\xi \mid)}-1}{\left(1+|\xi|^{2}\right)^{\frac{s}{2}}}\right| \leq \delta \gamma(|\xi|) \leq \delta \gamma(1) \lesssim \delta \leq \frac{1}{\left[\gamma^{-1}\left(\frac{\gamma(1)}{\delta}\right)\right]^{s}} .
$$

(ii) if $1<|\xi|<\gamma^{-1}\left(\frac{\gamma(1)}{\delta}\right)$, from the fact that $0<s \leq 1$ and $\frac{\gamma(t)}{t}: \mathbb{R}^{+} \rightarrow \mathbb{R}^{+}$is smooth and increasing on $(0, \infty)$, which leads to $\frac{\gamma(t)}{t^{s}}=\frac{\gamma(t)}{t} t^{1-s}$ is also increasing on $(0, \infty)$ and further implies that

$$
\left|m_{\gamma}(\xi)\right| \leq \frac{\delta \gamma(|\xi|)}{|\xi|^{s}} \leq \delta \frac{\gamma\left[\gamma^{-1}\left(\frac{\gamma(1)}{\delta}\right)\right]}{\left[\gamma^{-1}\left(\frac{\gamma(1)}{\delta}\right)\right]^{s}}=\frac{\gamma(1)}{\left[\gamma^{-1}\left(\frac{\gamma(1)}{\delta}\right)\right]^{s}} \lesssim \frac{1}{\left[\gamma^{-1}\left(\frac{\gamma(1)}{\delta}\right)\right]^{s}}
$$

(iii) if $|\xi| \geq \gamma^{-1}\left(\frac{\gamma(1)}{\delta}\right)$, from $t^{s}: \mathbb{R}^{+} \rightarrow \mathbb{R}^{+}$is smooth and increasing on $(0, \infty)$, we have that

$$
\left|m_{\gamma}(\xi)\right| \lesssim \frac{1}{|\xi|^{s}} \leq \frac{1}{\left[\gamma^{-1}\left(\frac{\gamma(1)}{\delta}\right)\right]^{s}} .
$$

This completes the proof of Lemma 2.2.

In the proof of Theorem 1.7 it suffices to proof the Lemma 2.3

Lemma 2.3. Let $n \geq 1,0<s \leq 1,0<\delta<1, m_{\gamma, \mu}(\xi):=\frac{e^{i\left(\delta^{\beta} \mu \cdot \xi+\delta \gamma(\xi \mid)\right)}-1}{\left(1+|\xi|^{2}\right)^{\frac{5}{2}}}, \gamma(t)$ be the same as in Theorem 1.5 Then $\left\|m_{\gamma, \mu}\right\|_{L^{\infty}\left(\mathbb{R}^{n}\right)} \leq C \frac{1}{\left[\gamma^{-1}\left(\frac{\gamma(1)}{\delta}\right)\right]^{s}}$ when $\beta>1,\left\|m_{\gamma, \mu}\right\|_{L^{\infty}\left(\mathbb{R}^{n}\right)} \leq C \frac{\delta^{\beta-1}}{\left[\gamma^{-1}\left(\frac{\gamma(1)}{\delta}\right)\right]^{s}}$ when $\beta \leq 1$, where the constant $C$ does not depend on $\delta$.

Proof. Similarly to the proof of Lemma 2.2, the proof can also be divided into three situations. When $\beta>1$,

(i) if $0 \leq|\xi| \leq 1$, we can also get $\gamma^{-1}\left(\frac{\gamma(1)}{\delta}\right) \leq \frac{1}{\delta}$ and $\delta \leq \frac{1}{\left[\gamma^{-1}\left(\frac{\gamma(1)}{\delta}\right)\right]^{s}}$.

$$
\left|m_{\gamma, \mu}(\xi)\right|=\left|\frac{e^{i\left(\delta^{\beta} \mu \cdot \xi+\delta \gamma(|\xi|)\right)}-1}{\left(1+|\xi|^{2}\right)^{\frac{s}{2}}}\right| \leq \delta(|\xi|+\gamma(|\xi|)) \leq \delta(1+\gamma(1)) \lesssim \delta \leq \frac{1}{\left[\gamma^{-1}\left(\frac{\gamma(1)}{\delta}\right)\right]^{s}}
$$


(ii) if $1<|\xi|<\gamma^{-1}\left(\frac{\gamma(1)}{\delta}\right)$, from the fact that $\frac{\gamma(t)}{t^{s}}=\frac{\gamma(t)}{t} t^{1-s}$ is also increasing on $(0, \infty)$ with $0<s \leq 1$ and further implies that

$$
\begin{aligned}
\left|m_{\gamma, \mu}(\xi)\right| & \leq \frac{\delta^{\beta}|\xi|+\delta \gamma(|\xi|)}{|\xi|^{s}} \\
& \leq \frac{\delta(|\xi|+\gamma(|\xi|))}{|\xi|^{s}} \\
& =\delta\left(|\xi|^{1-s}+\frac{\gamma(|\xi|)}{|\xi|^{s}}\right) \\
& \leq \delta\left(\left[\gamma^{-1}\left(\frac{\gamma(1)}{\delta}\right)\right]^{1-s}+\frac{\gamma\left[\gamma^{-1}\left(\frac{\gamma(1)}{\delta}\right)\right]}{\left[\gamma^{-1}\left(\frac{\gamma(1)}{\delta}\right)\right]^{s}}\right) \\
& =\delta\left[\gamma^{-1}\left(\frac{\gamma(1)}{\delta}\right)\right]^{1-s}+\frac{\gamma(1)}{\left[\gamma^{-1}\left(\frac{\gamma(1)}{\delta}\right)\right]^{s}} \\
& \lesssim \frac{1}{\left[\gamma^{-1}\left(\frac{\gamma(1)}{\delta}\right)\right]^{s}} .
\end{aligned}
$$

(iii) if $|\xi| \geq \gamma^{-1}\left(\frac{\gamma(1)}{\delta}\right)$, from $t^{s}: \mathbb{R}^{+} \rightarrow \mathbb{R}^{+}$is smooth and increasing on $(0, \infty)$, we have that

$$
\left|m_{\gamma, \mu}(\xi)\right| \lesssim \frac{1}{|\xi|^{s}} \leq \frac{1}{\left[\gamma^{-1}\left(\frac{\gamma(1)}{\delta}\right)\right]^{s}} .
$$

Next we consider the case of $\beta \leq 1$.

(i) if $0 \leq|\xi| \leq 1$, we can also get $\gamma^{-1}\left(\frac{\gamma(1)}{\delta}\right) \leq \frac{1}{\delta}$ and $\delta \leq \frac{1}{\left[\gamma^{-1}\left(\frac{\gamma(1)}{\delta}\right)\right]^{s}}$.

$$
\left|m_{\gamma, \mu}(\xi)\right|=\left|\frac{e^{i\left(\delta^{\beta} \mu \cdot \xi+\delta \gamma(|\xi|)\right)}-1}{\left(1+|\xi|^{2}\right)^{\frac{s}{2}}}\right| \leq \delta^{\beta}(|\xi|+\gamma(|\xi|)) \leq \delta^{\beta}(1+\gamma(1)) \lesssim \delta^{\beta} \lesssim \frac{\delta^{\beta-1}}{\left[\gamma^{-1}\left(\frac{\gamma(1)}{\delta}\right)\right]^{s}}
$$

(ii) if $1<|\xi|<\gamma^{-1}\left(\frac{\gamma(1)}{\delta}\right)$, from the fact that $\frac{\gamma(t)}{t^{s}}=\frac{\gamma(t)}{t} t^{1-s}$ is also increasing on $(0, \infty)$ with $0<s \leq 1$ and further implies that

$$
\begin{aligned}
\left|m_{\gamma, \mu}(\xi)\right| & \leq \frac{\delta^{\beta}|\xi|+\delta \gamma(|\xi|)}{|\xi|^{s}} \\
& \leq \frac{\delta^{\beta}(|\xi|+\gamma(|\xi|))}{|\xi|^{s}} \\
& =\delta^{\beta}\left(|\xi|^{1-s}+\frac{\gamma(|\xi|)}{|\xi|^{s}}\right) \\
& \leq \delta^{\beta}\left(\left[\gamma^{-1}\left(\frac{\gamma(1)}{\delta}\right)\right]^{1-s}+\frac{\gamma\left[\gamma^{-1}\left(\frac{\gamma(1)}{\delta}\right)\right]}{\left[\gamma^{-1}\left(\frac{\gamma(1)}{\delta}\right)\right]^{s}}\right) \\
& \lesssim \delta^{\beta}\left[\gamma^{-1}\left(\frac{\gamma(1)}{\delta}\right)\right]^{1-s}+\frac{\delta^{\beta-1}}{\left[\gamma^{-1}\left(\frac{\gamma(1)}{\delta}\right)\right]^{s}}
\end{aligned}
$$




$$
\lesssim \frac{\delta^{\beta-1}}{\left[\gamma^{-1}\left(\frac{\gamma(1)}{\delta}\right)\right]^{s}} .
$$

(iii) if $|\xi| \geq \gamma^{-1}\left(\frac{\gamma(1)}{\delta}\right)$, from $t^{s}: \mathbb{R}^{+} \rightarrow \mathbb{R}^{+}$is smooth and increasing on $(0, \infty)$, we have that

$$
\left|m_{\gamma, \mu}(\xi)\right| \lesssim \frac{1}{|\xi|^{s}} \leq \frac{1}{\left[\gamma^{-1}\left(\frac{\gamma(1)}{\delta}\right)\right]^{s}} \lesssim \frac{\delta^{\beta-1}}{\left[\gamma^{-1}\left(\frac{\gamma(1)}{\delta}\right)\right]^{s}} .
$$

This completes the proof of Lemma 2.3 .

\section{Acknowledgements}

The authors would like to thank Prof. Junfeng Li for many valuable comments and useful discussions.

\section{References}

[1] J. Bourgain, Some new estimates on oscillatory integrals, Essays on Fourier analysis in Honor of Elias M. Stein (Princeton, NJ, 1991), Princeton Math. Ser., vol. 42, Princeton University Press, New Jersey, 1995, pp. 83-112.

[2] J. Bourgain, On the Schrödinger maximal function in higher dimension, Proc. Steklov Inst. Math. 280 (2013), no. 1, 46-60.

[3] J. Bourgain, A note on the Schrödinger maximal function, J. Anal. Math. 130 (2016), 393396.

[4] L. Carleson, Some analytic problems related to statistical mechanics, in: Euclidean harmonic analysis (Proc. Sem., Univ. Maryland., College Park, Md., 1979), pages 5-45, Lecture Notes in Math., 779, Springer, Berlin, 1980.

[5] C. H. Cho and H. Ko, A note on maximal estimates of generalized Schrödinger equation, arXiv:1809.03246v1.

[6] C. H. Cho, S. Lee and A. Vargas, Problems on pointwise convergence of solutions to the Schrödinger equation, J. Fourier Anal. Appl. 18 (2012), no. 5, 972-994.

[7] B. E. J. Dahlberg and C. E. Kenig, A note on the almost everywhere behavior of solutions to the Schrödinger equation, in: Harmonic analysis (Minneapolis, Minn., 1981), pages 205209, Lecture Notes in Math., 908, Springer, Berlin-New York, 1982.

[8] Y. Ding and Y. Niu, Weighted maximal estimates along curve associated with dispersive equations, Anal. Appl. (Singap.) 15 (2017), no. 2, 225-240.

[9] X. Du, L. Guth and X. Li, A sharp Schrödinger maximal eatimate in $\mathbb{R}^{2}$, Ann. of Math. (2) 186 (2017), no. 2, 607-640.

[10] X. Du, L. Guth, X. Li and R. Zhang, Pointwise convergence of Schrödinger solutions and multilinear refined Strichartz estimates, Forum Math. Sigma 6 (2018), e14, 18 pp. Published online: doi:10.1017/fms.2018.11.

[11] X. Du, J. Kim, H. Wang and R. Zhang, Lower bounds for estimates of the Schrödinger maximal function, arXiv:1902.01430 
[12] X. Du and R. Zhang, Sharp $L^{2}$ estimate of Schrödinger maximal function in higher dimensions, Ann. of Math. (2) 189 (2019), no. 3, 837-861.

[13] S. Lee, On pointwise convergence of the solutions to Schrödinger equations in $\mathbb{R}^{2}$, Int. Math. Res. Not. 2006, Art. ID 32597, 21 pp.

[14] W. Li and H. Wang, Pointwise convergence of solutions to the Schrödinger equation along a class of curves, arXiv: $1807.00292 \mathrm{v} 1$.

[15] C. Miao, J. Yang and J. Zheng, An improved maximal inequality for 2D fractional order Schrödinger operators, Studia Math. 230 (2015), no. 2, 121-165.

[16] A. Moyua, A. Vargas and L. Vega, Schrödinger maximal function and restriction properties of the Fourier transform. Internat. Math. Res. Notices (1996), no. 16, 793-815.

[17] P. Sjögren and P. Sjölin, Convergence properties for the time-dependent Schrödinger equation, Ann. Acad. Sci. Fenn. Ser. A I Math. 14 (1989), no. 1, 13-25.

[18] P. Sjölin, Regularity of solutions to the Schrödinger equation, Duke Math. J. 55 (1987), no. 3, 699-715.

[19] P. Sjölin, Two theorems on convergence of Schrödinger means, J. Fourier Anal. Appl. http://doi.org/10.1007/s00041-018-9644-0.

[20] T. Tao and A. Vargas, A bilinear approach to cone multipliers. II. Applications, Geom. Funct. Anal. 10 (2000), no. 1, 216-258.

[21] L. Vega, E1 Multiplicador de Schrödinger, la Function Maximal y los Operadores de Restriccion(thesis). Departamento de Matematicas. Univ. Autónoma de Madrid, Madrid (1988).

[22] L. Vega, Schrödinger equations: pointwise convergence to the initial data, Proc. Amer. Math. Soc. 102 (1988), no. 4, 874-878.

Dan Li

Laboratory of Mathematics and Complex Systems (Ministry of Education of China), School of Mathematical Sciences, Beijing Normal University, Beijing 100875, People's Republic of China

E-mails: danli@mail.bnu.edu.cn

Haixia Yu (Corresponding author)

Department of Mathematics, Sun Yat-sen University, Guangzhou, 510275, People's Republic of China

E-mail: yuhaixia@mail.bnu.edu.cn 